\title{
Fabric-Based Active Electrode Design and Fabrication for Health Monitoring Clothing
}

\author{
Carey R. Merritt, H. Troy Nagle, Fellow, IEEE, and Edward Grant, Senior Member, IEEE
}

\begin{abstract}
In this paper, two versions of fabric-based active electrodes are presented to provide a wearable solution for ECG monitoring clothing. The first version of active electrode involved direct attachment of surface-mountable components to a textile screenprinted circuit using polymer thick film techniques. The second version involved attaching a much smaller, thinner, and less obtrusive interposer containing the active electrode circuitry to a simplified textile circuit. These designs explored techniques for electronic textile interconnection, chip attachment to textiles, and packaging of circuits on textiles for durability. The results from ECG tests indicate that the performance of each active electrode is comparable to commercial Ag/AgCl electrodes. The interposer-based active electrodes survived a five-cycle washing test while maintaining good signal integrity.
\end{abstract}

Index Terms-Active electrode, ECG, electronic textile, health monitoring clothing, nonwoven textile.

\section{INTRODUCTION}

$\mathbf{E}$ CG TECHNOLOGY requires the use of electrodes designed to measure the biopotentials emanating from the heart. Dry electrodes are highly desirable for long-term monitoring applications. Unlike the wet electrodes used for clinical monitoring, dry electrodes avoid the "drying out" phenomenon associated with the adhesives and gel membranes used with wet electrodes. Dry electrodes are generally biocompatible with the skin because they do not use adhesive or gel membranes. Such membranes are commonly used in commercial $\mathrm{Ag} / \mathrm{AgCl}$ electrodes and can cause serious skin irritation, including dermatitis. Because dry electrodes are well suited for long-term monitoring, they are used almost exclusively in electronic-textile-based health monitoring clothing that is available to date.

Dry electrodes do, however, suffer from noise interference. Noise, in this case, is typically generated from motion artifacts and power line interference. A common solution used to suppress noise in dry electrode signals is a buffer amplifier. A buffer amplifier is essentially an impedance converter, one that converts high-impedance signals to low-impedance signals [1]. Now, whereas, high-impedance signals are very susceptible to both noise and motion-induced artifacts in signal paths, a lowimpedance signal is virtually unaffected by either of these artifacts [2]. As a result, implementing a simple buffer amplifier

Manuscript received July 25, 2008. First published January 23, 2009; current version published March 3, 2009.

C. R. Merritt and E. Grant are with the Department of Electrical and Computer Engineering and the University of North Carolina (UNC)-North Carolina State University (NCSU) Department of Biomedical Engineering, NCSU, Raleigh, NC 27695 USA (e-mail: carey.merritt@gmail.com; egrant@ ncsu.edu).

H. T. Nagle is with the University of North Carolina (UNC)-North Carolina State University (NCSU) Department of Biomedical Engineering, NCSU, Raleigh, NC 27695 USA (e-mail: nagle@unc.edu).

Digital Object Identifier 10.1109/TITB.2009.2012408

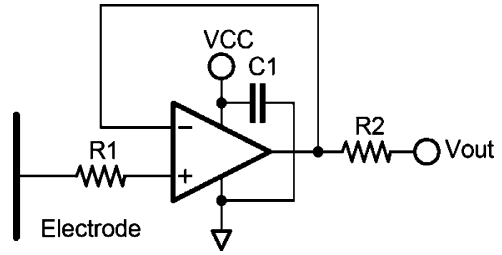

Fig. 1. Active electrode schematic.

at the electrode site reduces the signal impedance and prevents noise generation. Dry electrodes are also susceptible to noise interference because they typically have higher skin-electrode contact impedances than wet electrodes. Again, by introducing a buffer amplifier at the electrode site, the electrode source impedance, as seen by downstream electronic circuits, will be reduced. This produces an associated reduction in the intensity of the impedance mismatch between electrodes, which is known to produce a differential common-mode voltage that can easily saturate the instrumentation circuitry, and thereby, render the recorded signals useless [3].

Recent research has experimented with integrating buffer amplifiers with electronic textiles to produce fabric-based active electrodes [4]. Fabric-based active electrodes are produced by screen printing a buffer circuit pattern and electrode onto a nonwoven textile. Thereafter, the electrical components are directly attached to the fabric circuit to produce an active electrode. Early results from this research showed promise toward developing a resilient active electrode for utilization in a health monitoring garment. This paper reports continued active electrode research that was presented in [4], and also presents an update on the previous design, introduces a smaller, thinner, and less obtrusive design, and examines the durability and performance of the two fabric-based active electrode designs.

\section{DESIGN AND FABRICATION}

The fabric-based active electrode is a continuation of research presented in [4]. The design is based on a voltage follower circuit implemented with a few discrete components. The circuit, shown in Fig. 1, is a voltage follower that utilizes a currentlimiting resistor $R_{1}$ on the positive input, a power-decoupling capacitor $C_{1}$, and a resistor $R_{2}$ on the output. The values used for these components are $200 \mathrm{k} \Omega, 10 \mathrm{nF}$, and $100 \Omega$, respectively. The electrode is connected to the input resistor $R_{1}$.

Two versions of the fabric-based active electrode were designed and fabricated in this research. The first version involved screen printing the circuit onto a nonwoven textile and directly attaching the surface mount device (SMD) components to the textile circuit. The second version explored the possibility of 


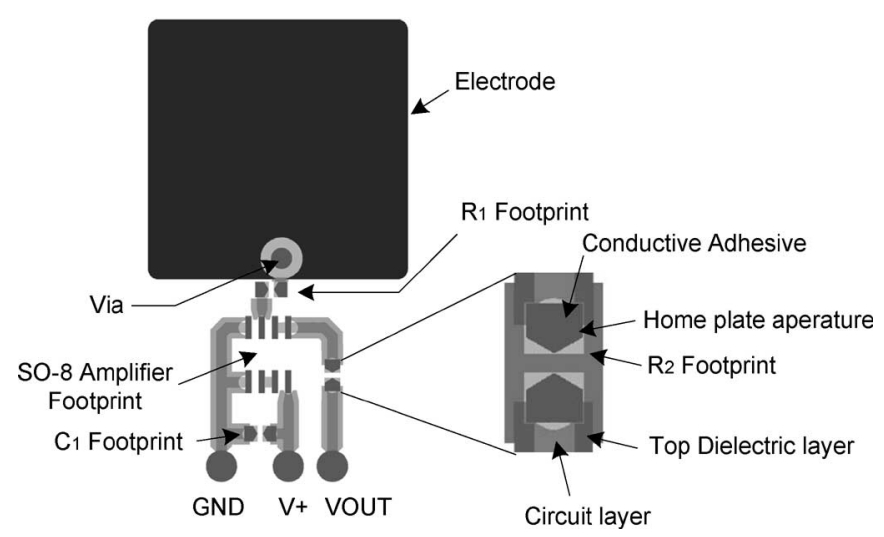

Fig. 2. Active electrode screen/stencil design using home plate pattern.

using an interposer, thereby allowing placement of the circuit and its components on a much smaller printed circuit board (PCB). The use of an interposer offers significant advantages in regards to size, cost, manufacturability, and resilience.

\section{A. Direct-Attach Active Electrodes}

The first active electrode design involves directly attaching SMD components to a screen-printed electronic textile circuit. This design, first presented in [4], is updated with a durable carbon-rubber electrode and modified encapsulation pattern to promote durability.

1) Design: The direct-attach design comprises several layers: electrode, circuit, dielectric coating, and conductive adhesive (see Fig. 2). The electrode layer is located on the reverse side of the fabric, and it is connected to the circuit on the opposite side of the fabric by a via positioned next to the resistor $R_{1}$.

The screen artwork for the active electrode circuit was designed to overcome the many production issues faced in printing on nonwoven textiles. For example, nonwoven fabrics are produced by water jets that extrude small fibers across a flat surface. This process can generate visible jet streaks of fibers across the surface of the substrate. Hence, in this design, the majority of the conductive lines are oriented in the machine direction because printing across the fiber streaks inherent in the fabric gives less than desirable results. Also, the direction recommended for the screen printer's squeegee to travel in is the direction of the longest conductive lines in the circuit's design [5]. In addition, the conductive lines are printed with a width of $1 \mathrm{~mm}$ to reduce the percentage of voids that can hamper line conductivity. Furthermore, the circuit is positioned away from the top of the electrode to allow the electrode to conform to the contours of the body and to reduce the risk of short circuits to the electrode, e.g., from pinholes in the fabric.

A dielectric layer was included in the active electrode circuit to serve as a protective barrier for the conductive traces and to prevent the traces from developing any microfractures that can lead to open circuits. The dielectric pattern was extended $0.5 \mathrm{~mm}$ on each side of the conductive traces to provide a $50 \%$ margin of protection.
The conductive adhesive stencil design, shown in Fig. 2, includes patterns for SMD pads, external pads, and the electrodeto- $R_{1}$ via. The apertures for the chip components were designed using the "home plate" pattern that was suggested in [6] to prevent shorts from forming underneath the components. The stencil design, along with the other layers, also includes four fiducials that are located on the outside perimeter, not shown in image. These fiducials were used to assist in screen alignment or re-registration for printing multiple layers, e.g., circuit, multiple prints of dielectric, and stenciling the conductive adhesive.

2) Fabrication: The screen printing parameters that were used to fabricate the active electrodes are summarized in [4]. Initially, the parameters used for printing the electrode layer in the preliminary experiments were the same as that for printing the circuit layer, e.g., a 200 mesh count. However, the ink coverage was insufficient, and this resulted in voids and an inconsistent printed circuit. A general observation made during screen printing was that larger areas, like the surface of the electrode, do not print as well as conductive lines when the same screen parameters are used. In order to increase the ink coverage or layer thickness, the mesh count was decreased, thus resulting in an increase in the total wet ink volume delivered during printing. A mesh count of 105 threads per inch was found suitable for printing the electrode onto the nonwoven material, Evolon.

The dielectric layer was printed using an 80 mesh count screen. Preliminary tests with Creative Materials (CMI) 11530 ink, using a higher mesh count screen, resulted in a poor conformal surface that left voids and pinholes. Therefore, the screen's mesh count was reduced in order to print a larger volume of ink. As a result, only two to three print cycles were required for printing the dielectric topcoat, which is typical for polymer thick film (PTF) dielectrics [5]. The active electrodes presented in this paper were fabricated using three prints of dielectric with a curing step applied between each print.

The active electrodes were fabricated using the PTF processing techniques described in [5]. The thick-film processes used for fabricating the active electrodes were: screen printing, stenciling, curing, and encapsulation. The encapsulation step involved testing the two solid black patterns that are defined in Fig. 3. The pattern in Fig. 3(b), inspired by [7], is an attempt to provide stress relief on the printed traces at the border of the rigid encapsulant. Semicircular stress relief areas were incorporated into the design to prevent the line on the rigid encapsulant perimeter from flexing and cracking. These encapsulant patterns were produced by creating an adhesive mask for each pattern out of the transparency film. After dispensing the encapsulant over the desired areas, the mask was carefully peeled back to yield the patterns shown in Fig. 3.

The steps required for active electrode fabrication are listed next and the final fabricated active electrode is shown in Fig. 4.

1) Print electrode on fabric backside with CMI 112-15 and cure in dryer at $100{ }^{\circ} \mathrm{C}$.

2) Print circuit on fabric topside with CMI 112-15 and cure in dryer at $100{ }^{\circ} \mathrm{C}$.

3) Print dielectric over circuit with CMI 115-30 and cure in dryer at $100{ }^{\circ} \mathrm{C}$, and repeat three times. 


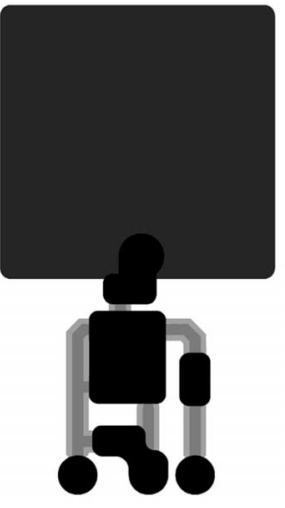

(a)

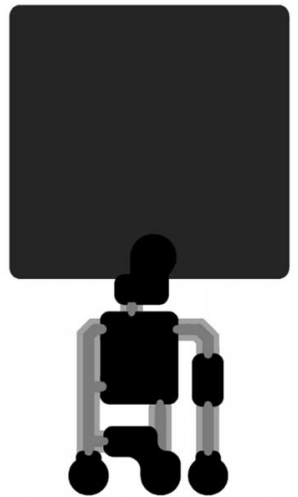

(b)
Fig. 3. Encapsulation patterns. (a) Regular pattern. (b) Stress relief pattern.

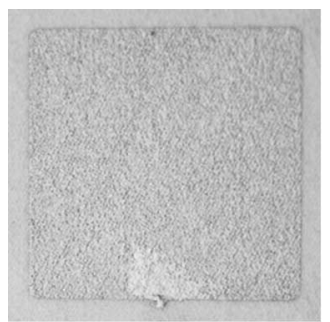

(a)

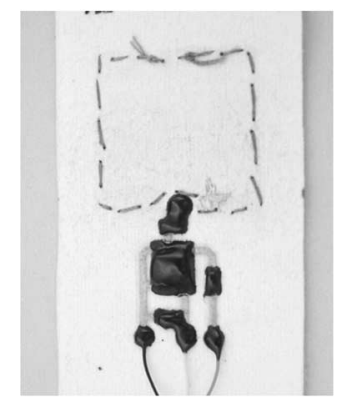

(c)

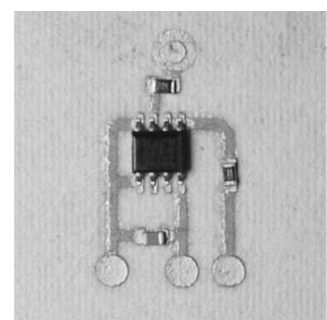

(b)

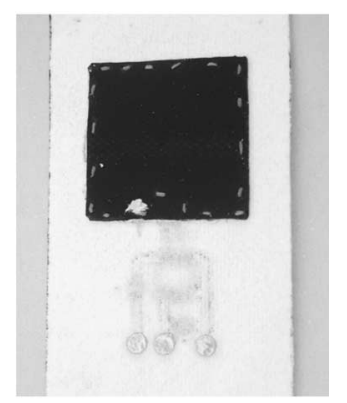

(d)
Fig. 4. Fabricated direct-attach active electrode. (a) Screen-printed electrode (bottom view). (b) Assembled circuit (top view). (c) Active electrode with encapsulated components (top view). (d) Active electrode with carbon-rubber electrode added (bottom view)

4) Drill or punch via hole to electrode.

5) Stencil conductive adhesive (CMI 119-05 and CMI 11944 mixed at 100:2.3) and attach components and cure in oven for $1 \mathrm{~h}$ at $130{ }^{\circ} \mathrm{C}$.

6) Encapsulate components with Hysol FP4460 using a 18gauge tip and cure for $4 \mathrm{~h}$ at $130{ }^{\circ} \mathrm{C}$.

7) Bond carbon-rubber electrodes to screen-printed electrode using ECCOSHIELD RVS and stitch the electrodes to the fabric.

\section{B. Interposer Active Electrodes}

Active electrodes were also designed and fabricated using an interposer approach. Interposers range from adapter boards that convert package technologies, e.g., SO-8 to DIP-8, to complex multichip modules that contain entire systems. In our case, the

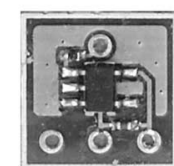

(a)

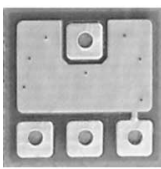

(b)

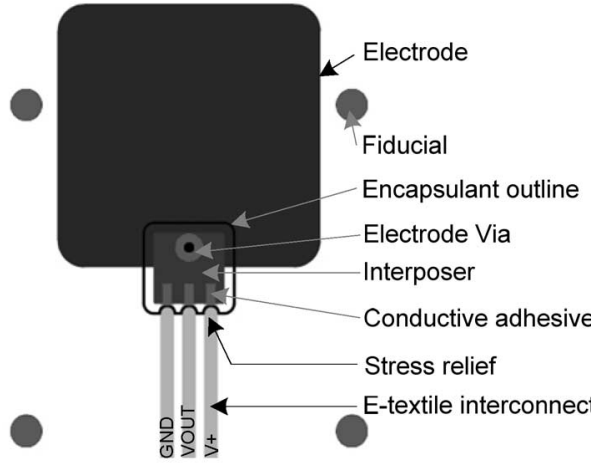

(c)
Fig. 5. Picture of interposer design. (a) Top view. (b) Bottom view. (c) Active electrode design for interposer approach.

interposer was used to connect SMD devices to an electronic textile circuit having somewhat larger feature size limitations than SMDs.

There are several benefits to using an interposer for attaching electronics to textiles, especially when compared to the earlier direct-attachment design. Most of the processing for an electronic textile using an interposer is accomplished on a PCB of either flexible or rigid FR-4 materials. As a result, high temperatures can be used so that the components can be attached by soldering, and they can be placed by using automated pick and place assembly machines. Also, using a PCB as an interposer permits smaller feature sizes, e.g., line widths and spacing down to $0.002 \mathrm{in}$, thereby allowing smaller component selection and smaller circuit areas. Thus, greater circuit complexity can be packed into a smaller area. Furthermore, an interposer enables custom pad designs where the same footprint will work with a variety of electronic textile technologies. For example, in [8] they designed a flexible circuit interposer for recording capacitive-based electromyography signals. In this instance, the perimeter pads were large enough so that the conductive interconnects could be embroidered directly through each pad. Another benefit to using interposers relates to circuit failure. Using an interposer simplifies the electronic textile circuit, thereby reducing the chance of circuit failure through cracked lines or broken bonds between the electronic textile and SMD components.

1) Design: The interposer active electrode design uses the same circuit as the direct-attach version, but with a few modifications. The resistor $R_{1}$ substitutes a $100 \mathrm{k} \Omega$ resistor and the resistor $R_{2}$ is removed from the design. Also, the amplifier is replaced with an OPA333 to take advantage of its small package size (SC-70), low noise, and micropower capabilities (17 $\mu \mathrm{A})$.

The PCB design is shown in Fig. 5(a) and (b). The bottom pads are spaced $2.08 \mathrm{~mm}$ apart and are $1.50 \mathrm{~mm}$ wide. The PCB material is 0.005 -in-thick FR-4 with $0.5 \mathrm{oz}$ copper cladding and a gold finish. The board dimension is $6.5 \mathrm{~mm} \times 6.5 \mathrm{~mm}$, which is a little larger in area than the SO- 8 amplifier that was used in the direct-attach design. The board design utilized an active guard to reduce any stray capacitance that could couple to the sensitive, high-impedance input signal line. An active guard was 


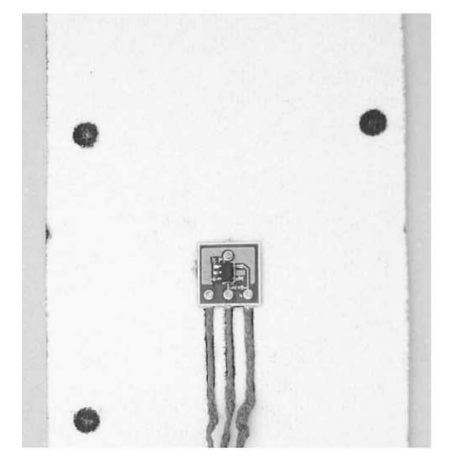

(a)

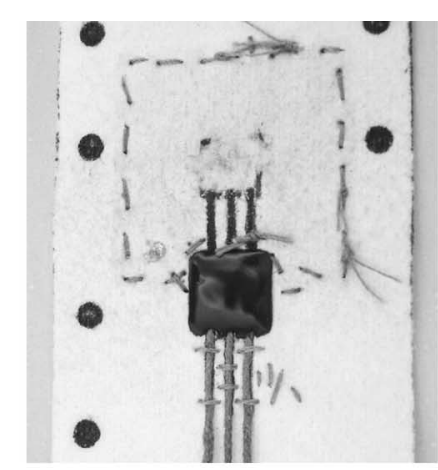

(b)

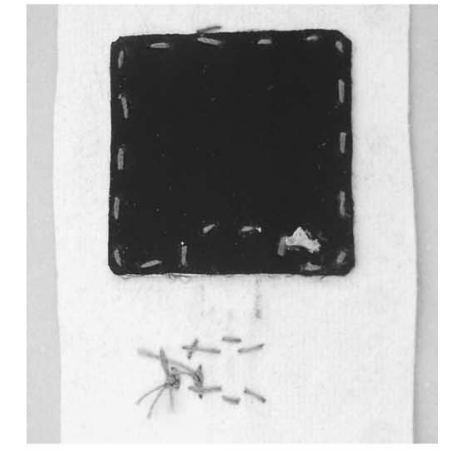

(c)

Fig. 6. Picture of fabricated interposer active electrode. (a) Interposer attached to fabric after step five. (b) Finished active electrode (top view). (c) Finished active electrode (bottom view).

implemented by substituting it in place of the top and bottom ground plane.

The active electrode design, including the interposer, is shown in Fig. 5(c). The electrode remains on the bottom side of the fabric to make contact with the body, while the buffer resides on the top side. The input pad of the interposer is connected to the electrode by a via that is filled with conductive adhesive.-Fig. 5(c) depicts the multiple layers involved in the design and shows the four round fiducials that were added to aid in the alignment of each layer during the fabrication process. The encapsulant pattern is shown as a black outline surrounding the interposer [see Fig. 5(c)]. This pattern uses the stress relief concept introduced in the direct-attach approach to prevent stress fractures in the conductive interconnects at the encapsulant border.

2) Fabrication: The interposer board design enables a greater selection of fabrication techniques for producing the active electrodes. The board design includes large perimeter pads that are spaced sufficiently to accommodate screen printing and other established electronic textile technologies, like embroidery. In addition, the via's on each pad permit hand stitching the interposer to a garment with either a conductive yarn or thread. In this research, the same screen printing methods that were applied in the direct-attach design were used to implement the interposer design. As a result, the fabrication steps are consistent except that a single populated interposer, which contains the entire active electrode circuit, is attached as opposed to individual SMD's. However, the active electrodes for this part of the study were custom built by hand to validate the design prior to considering methods for mass producing the electrodes.

The fabrication steps for these active electrodes were modified slightly in order to prototype the design. For example, conductive yarns were used in place of the screen-printed interconnects (signal, power, and ground lines). The conductive yarn that was used for the textile interconnections was extracted from commercially available conductive elastic made by Textronics, Inc., by delaminating the nonwoven material with a temperature of approximately $120{ }^{\circ} \mathrm{C}$. Since the conductive yarn was washable, no protective dielectric was required for the electronic textile circuit, thus removing the need for one additional step. Despite using conductive yarn in place of screen printing for the interconnections, the majority of the fabrication steps are still the same as the direct-attach version. The steps for fabrication are best described in the list that follows.

1) Screen print electrode on fabric backside.

2) Mark fiducials, three interconnections, and outline for interposer placement.

3) Embroider conductive yarn electronic textile circuit.

4) Drill or punch via to electrode.

5) Stencil conductive epoxy (CircuitWorks CW2400) and attach populated interposer.

6) Encapsulate interposer with glob top encapsulant.

7) Bond and stitch commercial carbon-rubber electrodes to screen-printed electrode.

The interposer active electrodes that were fabricated for this research are shown in Fig. 6. The conductive adhesive was applied by stenciling conductive epoxy over the conductive yarn and electrode via by using an adhesive stencil. Initially, shorts occurred among the three interposer pads, but this problem was solved by reducing the stencil's aperture width for each pad to $56 \%$ of the original pad width. A mask was also designed to control the encapsulation pattern. This pattern is shown in Fig. 5(c) as the dark outline surrounding the interposer with a $1.5 \mathrm{~mm}$ margin. The resulting average thickness of the encapsulant was around $1.70 \mathrm{~mm}$ compared to the direct-attach thickness of $2.45 \mathrm{~mm}$. The size of the entire encapsulated interposer was $11.6 \mathrm{~mm} \times 11.6 \mathrm{~mm}$ compared to the approximate $20 \mathrm{~mm} \times 13 \mathrm{~mm}$ size of the direct-attach version. Thus, the area and thickness of the interposer are significantly reduced by $48 \%$ and $31 \%$, respectively.

\section{EXPERIMENTAL RESULTS}

Experiments were designed to compare the performance of new and novel textile-based active electrode designs to a passive carbon electrode and a commercially available $\mathrm{Ag} / \mathrm{AgCl}$ electrode. A series of experiments were conducted to evaluate the durability and reliability of these new active electrodes for potential use in health monitoring garments. The active electrodes were evaluated by recording ECG signals, and examining the time and frequency behavior of the measured signals. 


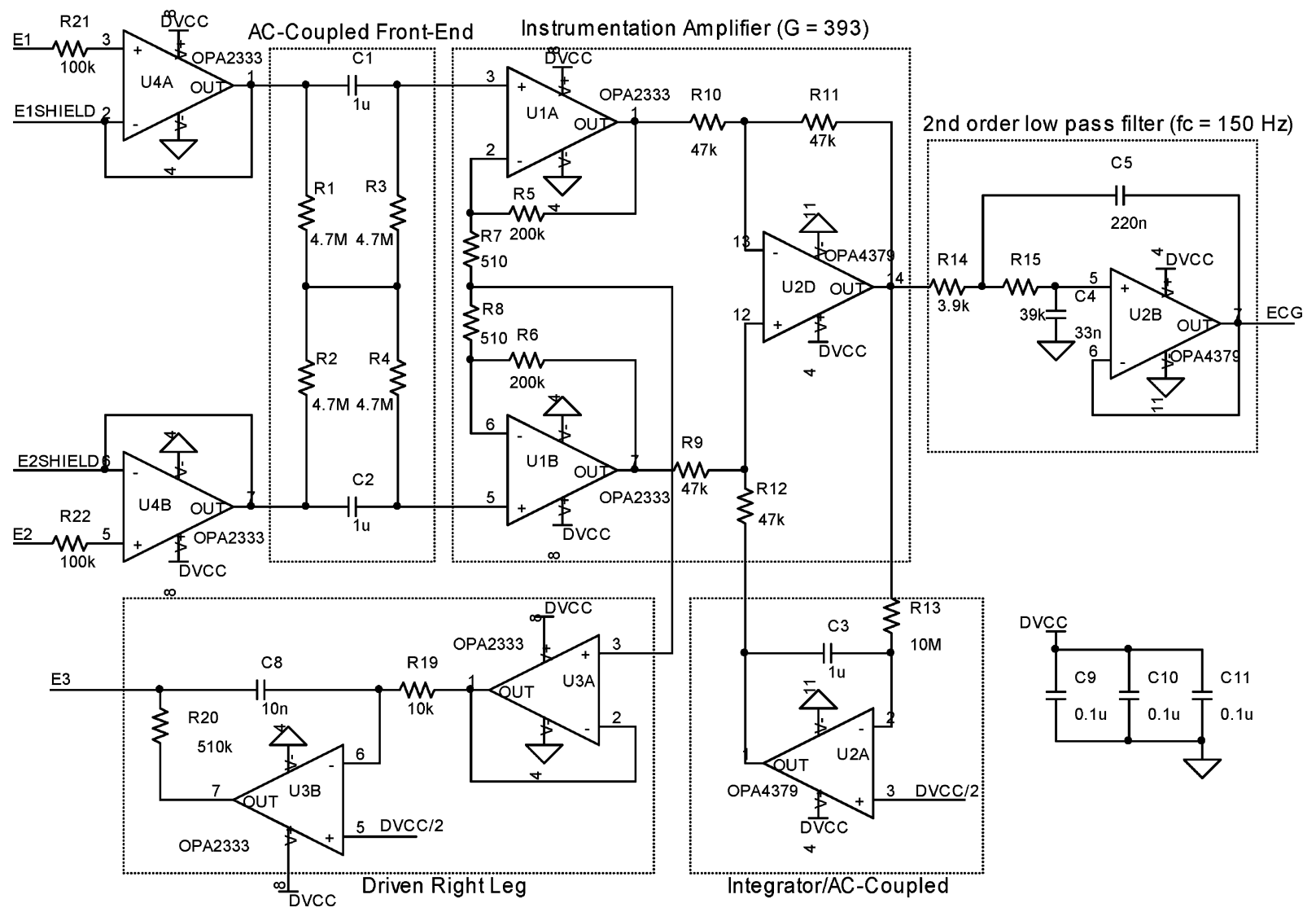

Fig. 7. Single supply ECG circuit for testing active electrodes.

\section{A. Experimental Setup}

The circuit that was used to measure ECG was modified from the circuit proposed in [9]. The ECG circuit, Fig. 7, is a single supply ac-coupled biopotential amplifier customized for recording the data in this research. The amplifier was designed with a gain of 393 and filter cutoff frequencies $F_{\mathrm{HP}}=0.04 \mathrm{~Hz}$ and $F_{\mathrm{LP}}=150 \mathrm{~Hz}$ for the associated highpass and low-pass filters, respectively. The analog ECG signal was recorded at 1000 samples per second with a 10-bit ADC on the Texas Instruments MSP430F2274 microcontroller and serially transmitted to a PC running MATLAB for data collection.

The passive electrodes were connected to the circuit with shielded cables; the shielding was connected to the buffered output of the electrode. The shielding was necessary to remove most of the cable noise and permit a direct comparison of the active and passive electrodes. The active electrodes were connected to the circuit by bypassing the $100 \mathrm{k} \Omega$ resistors, $R_{21}$ and $R_{22}$.

Each electrode type was tested for 5 min while sitting, and 5 min while jogging in place. These recordings occurred $30 \mathrm{~min}$ after the electrodes were positioned on the body to allow the skin-electrode impedance to stabilize. For each experiment, two electrodes (E1 and E2) were held firmly in place on the subject's thorax by an elastic belt, and a commercial $\mathrm{Ag} / \mathrm{AgCl}$ electrode was placed on the subject's waist to serve as the driven right leg electrode (E3). The following electrodes were tested:

1) three direct-attach active electrodes;

2) three direct-attach active electrodes with stress relief encapsulant pattern;

3) three interposer active electrodes;

4) three carbon-rubber passive electrodes;

5) one pair of commercial $\mathrm{Ag} / \mathrm{AgCl}$ passive electrodes (Cleartrace REF1700 by CONMED Corporation).

\section{B. Comparative Analysis (Before Washing)}

The combined experimental data from the ECG experiments using the fabricated electrodes are shown in Fig. 8. The data presented in this figure are a sample of 5-min recording sessions, starting at $200 \mathrm{~s}$, and includes data for both sitting and jogging. The data presented here suggest that there are no significant differences between the new electrodes under test and the commercially available $\mathrm{Ag} / \mathrm{AgCl}$ electrodes. Further testing is planned to quantify the long-term usefulness of these new electrodes for monitoring clothing.

The jogging data demonstrated that the active electrodes were able to effectively operate as impedance converters and to suppress line noise. Surprisingly, the high-frequency noise is only slightly elevated when compared to the data recorded for sitting. The clear QRS complex peaks in the jogging data suggest that the active electrodes will enable parameter extraction during exercise, like heart rate, with greater accuracy and with fewer false positives. 
(a)

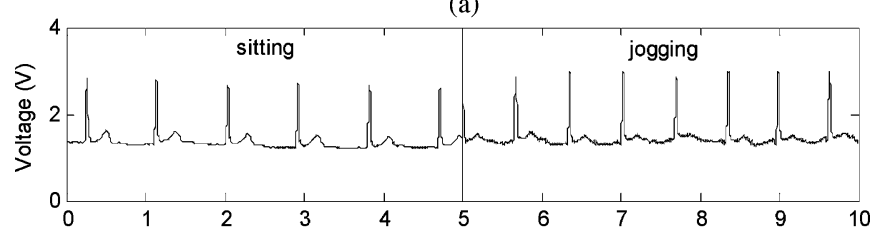

(b)

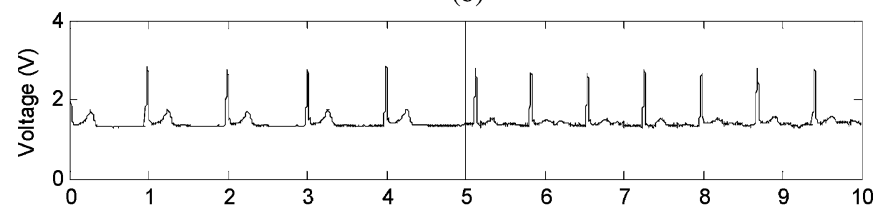

(c)

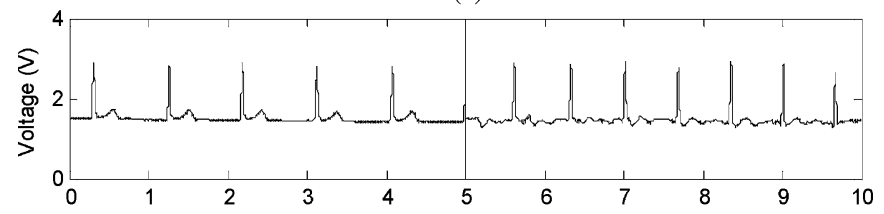

(d)

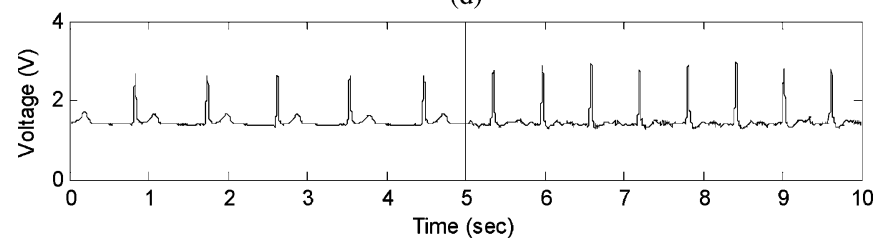

Fig. 8. Combined ECG recordings while sitting $(5 \mathrm{~s})$ and jogging $(5 \mathrm{~s})$ for four types of electrodes. (a) Direct-attach active electrode. (b) Interposer active electrode. (c) Carbon-rubber electrode. (d) Commercial $\mathrm{Ag} / \mathrm{AgCl}$ electrode.

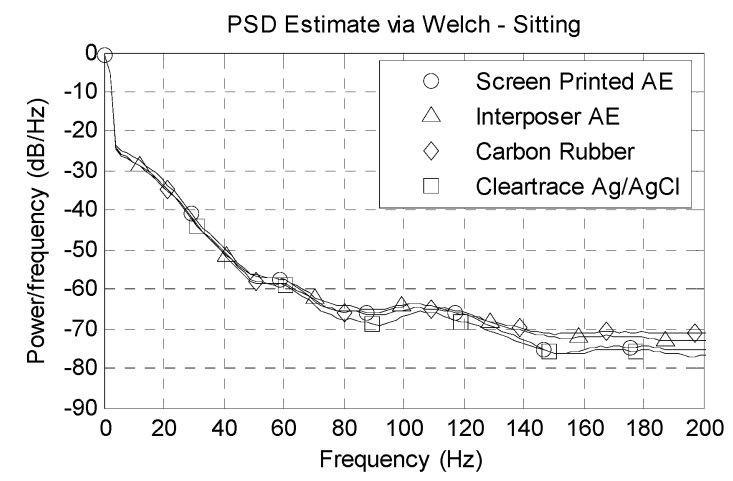

(a)

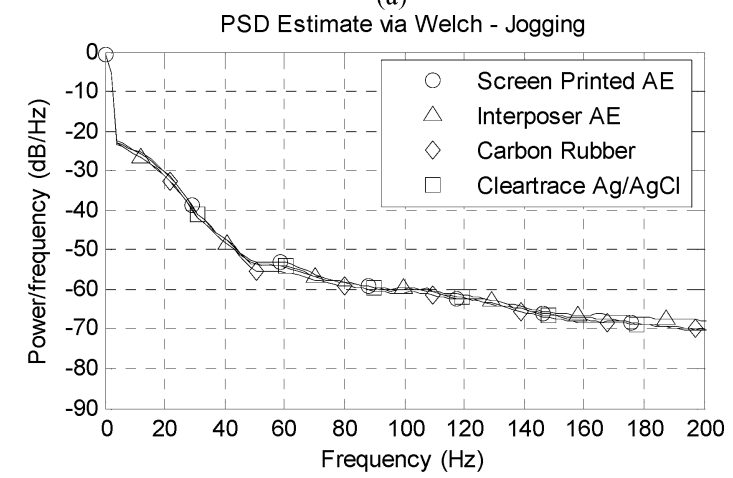

(b)

Fig. 9. PSD estimate for (a) sitting and (b) jogging in place.

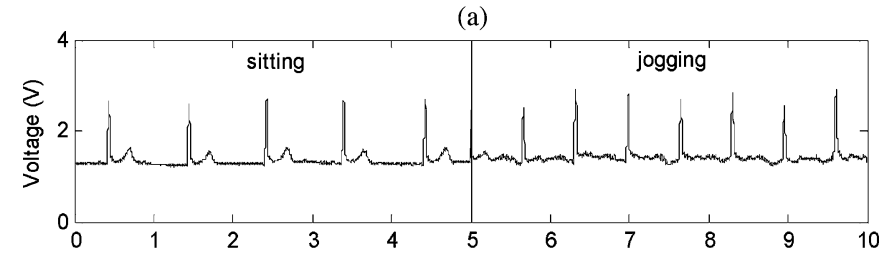

(b)

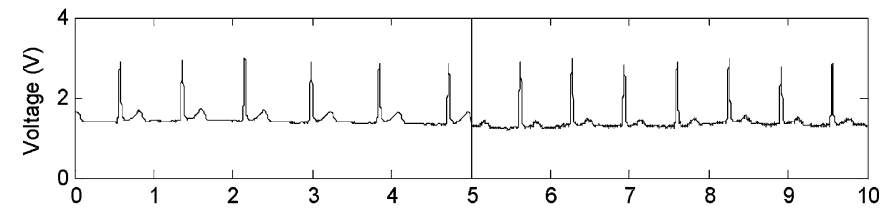

(c)

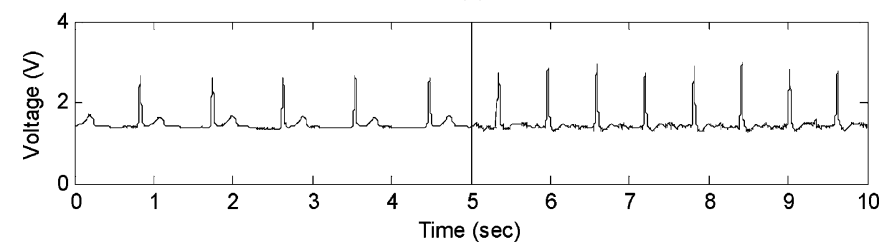

Fig. 10. Combined ECG recordings while sitting (5 s) and jogging (5 s) after washing electrodes five times with exception of commercial electrode. (a) Interposer active electrode. (b) Carbon-rubber electrode. (c) Commercial $\mathrm{Ag} / \mathrm{AgCl}$ electrode.

The 5-min dataset recorded from these experiments was also evaluated in the frequency domain, using a power spectral density (PSD) estimate. The PSD was estimated using the MATLAB "pwelch" method that is part of the Signal Processing Toolbox. This method returns the power spectral density estimate on a dataset by using Welch's averaged, modified periodogram method. Here, a 512-sample size window was used with the default 50\% overlap to compute the one-sided PSD for each ECG recording. The PSDs for each electrode type (three trials for each type) were averaged and plotted in Fig. 9 for sitting and jogging. The PSDs for sitting and jogging indicate that the active electrodes perform closely to the $\mathrm{Ag} / \mathrm{AgCl}$ electrode.

\section{Durability Analysis (After Washing)}

Durability testing was performed by machine washing the electrode samples for five cycles with exception of the commercial electrodes. The washing cycles involved running the washer on the permanent press setting and air drying the samples afterward. The samples were hand stitched to larger sheets of Evolon so that they were properly washed as if they were attached to a garment. The passive carbon-rubber samples were also included in the same washing tests to help sort out problems that are related to the electrode versus the circuit.

Once the electrodes were washed for the complete five cycles, they were tested by passing a sine wave through each electrode. The signal was applied using a metal pad $(30 \mathrm{~mm} \times 30 \mathrm{~mm})$ in contact with the electrode's surface. The direct-attach active electrodes did not function due to a crack formed in the line from $R_{1}$ to the amplifier's positive input. There were also cracks in other areas, especially on the samples without stress relief but they did not prevent the circuit from passing the sine-wave test. No further testing was performed on the direct-attach electrodes 
for this experiment since they would require a design change to reduce the stress on the signal line between $R_{1}$ and the amplifier.

The interposer active electrode and the passive carbon electrodes passed the sine-wave test after five washes with no exceptions, so they were finally tested on the thorax of a human subject. The same ECG experiments were performed on the electrodes, and the combined sitting and jogging ECG signals are displayed in Fig. 10. The results in Fig. 10 indicate that there are no major differences between before (Fig. 8) and after washing (Fig. 10). The signals still have good clarity and visually compare well to the commercial $\mathrm{Ag} / \mathrm{AgCl}$ electrode.

\section{CONCLUSION}

This research examined the use of two versions of fabricbased active electrodes to improve the performance of dry electrodes used in monitoring clothing. These two designs dealt with issues related to integrating electronics into clothing and durability.

The experimental results on the active electrodes showed that they compare well to commercial wet $\mathrm{Ag} / \mathrm{AgCl}$ electrodes. During jogging tests, the active electrodes demonstrated strong visual QRS complex peaks and stable signals with relatively little motion artifact. In addition, the durability of each active electrode type was demonstrated by using the five-cycle washing test. These washing tests revealed problems with the directattach design, while the interposer design showed successful and clean operation after washing. The interposer design maintained signal fidelities comparable to commercial $\mathrm{Ag} / \mathrm{AgCl}$ electrodes both before and after washing. While more extensive trials are required to determine their longevity, the success of the much smaller interposer design suggests that the interposer style active electrode is the preferred solution for implementation into health monitoring clothing.

\section{REFERENCES}

[1] E. S. Valchinov and N. E. Pallikarakis. (2004). An active electrode for biopotential recording from small localized bio-sources. Biomed. Eng. Online [Online] 3(25). Available: http://www.biomedical-engineeringonline. com/content/pdf/1475-925X-3-25.pdf

[2] S. Nishimura, Y. Tomita, and T. Horiuchi, "Clinical application of an active electrode using an operational amplifier," IEEE Trans. Biomed. Eng., vol. 39, no. 10, pp. 1096-1099, Oct. 1992.

[3] A. Searle and L. Kirkup. (2000). A direct comparison of wet, dry and insulating bioelectric recording electrodes. Physiol. Meas. [Online] 21(2), pp. 271-283. Available: http://stacks.iop.org/0967-3334/21/271

[4] T.-H. Kang, C. R. Merritt, E. Grant, B. Pourdeyhimi, and H. T. Nagle, "Nonwoven fabric active electrodes for biopotential measurement during normal daily activity," IEEE Trans. Biomed. Eng., vol. 55, no. 1, pp. 188195, Jan. 2008.

[5] K. Gilleo, Polymer Thick Film. New York: Van Nostrand, 1996.

[6] IPC-7525, Stencil Design Guidelines. Bannockburn, IL: IPC, 2000.

[7] Acheson, Eliminate Silver Ink Cracking. (2008). [Online]. Available: http:// achesonelectronicmaterials.com/doc/guides/USA/Silver Cracking.pdf.

[8] T. Linz, L. Gourmelon, and G. Langereis, "Contactless EMG sensors embroidered onto textile," presented at the IFMBE 4th Int. Workshop Wearable Implantable Body Sensor Netw. (BSN 2007), RWTH Aachen Univ., Aachen, Germany.

[9] E. M. Spinelli, R. Pallas-Areny, and M. A. Mayosky, "AC-coupled frontend for biopotential measurements," IEEE Trans. Biomed. Eng., vol. 50, no. 3, pp. 391-395, Mar. 2003.

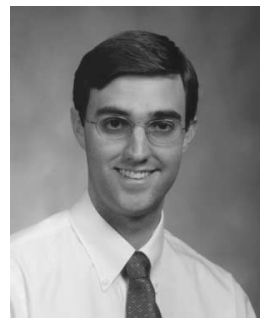

Carey R. Merritt received the B.Sc. and M.Sc. degrees in computer engineering, and the $\mathrm{Ph} . \mathrm{D}$. degree in electrical engineering with a comajor in biomedical engineering from North Carolina State University (NC State), Raleigh, in 2001, 2003, and 2008, respectively.

He was a Researcher in the Center for Robotics and Intelligent Machines (CRIM), North Carolina State University. His current research interests include medical instrumentation, embedded systems, and biomedical sensors.

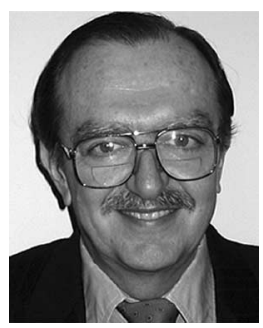

H. Troy Nagle (S'66-M'70-SM'74-F'83) received the B.S.E.E. and M.S.E.E. degrees from the University of Alabama, Tuscaloosa, the Ph.D. degree in electrical engineering from Auburn University, Auburn, AL, and the M.D. degree from the University of Miami School of Medicine, Miami, FL.

He is currently a Professor and a Founding Chair of the Joint University of North Carolina Chapel Hill/North Carolina State University Department of Biomedical Engineering, and a Professor of electrical and computer engineering at North Carolina State University, Raleigh, where he is the Director of the Biomedical Instrumentation Laboratory, a facility for prototyping medical devices. He has widely published in data acquisition and signal processing, and is coauthor of textbooks in digital logic design and sampled-data-control systems. Over the last several years, he has developed an electronic nose prototype and experimented with its use in food processing, environmental monitoring, and medical diagnostics. He is the coeditor of a handbook on machine olfaction published by Wiley VCH.

Dr. Nagle is a member of Phi Kappa Phi, Tau Beta Pi, Eta Kappa Nu, Sigma Xi, and Omicron Delta Kappa, and a Fellow of the American Institute for Medical and Biological Engineering (AIMBE). He was the recipient of the IEEE Richard M. Emberson Award for outstanding service to the Institute in Technical Activities. He was the President of the IEEE Industrial Electronics Society (IES) during 1984-1985, the Chairman of the IEEE Neural Networks Committee in 1988, and the IEEE Vice President for Technical Activities during 1989-1990. He was the IEEE President in 1994, the Chair of the IEEE Awards Board during 2000-2001, and the Chair of the IEEE Medal of Honor Selection Committee during 2002-2003. He is currently a member of the IEEE Sensors Council and the Editor-in-Chief of the IEEE SENSORS JOURNAL. He is a Registered Professional Engineer.

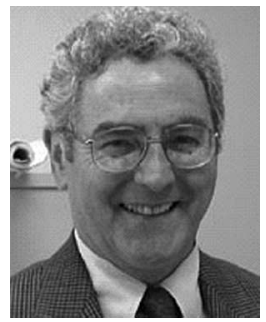

Edward Grant (M'88-SM'00) received the B.S. (Hons.) degree in mechanical engineering from Dundee College of Technology (now University of Abertay Dundee), Scotland, U.K., in 1969, the M.Eng. degree in mechanical engineering (fluid power control) from the University of Sheffield, Sheffield, U.K., in 1972, and the Ph.D. degree in computer science from the University of Strathclyde, Glasgow, U.K., in 2000.

$\mathrm{He}$ is currently the Director of the Center for Robotics and Intelligent Machines (CRIM), North Carolina State University (NC State), Raleigh, where he is a Professor of electrical and computer engineering at NC State and a Professor in the Joint Department of Biomedical Engineering for University of North Carolina (UNC)Chapel Hill and NC State. His current research interests include evolutionary robotics, robotics applied to rehabilitation engineering, large area distributed sensor systems, wearable computing, and biorobotics.

Dr. Grant is a Fellow of the Institution of Mechanical Engineers. During 1992, he was the General Chair of the International Symposium on Intelligent Control. From 1993 to 1997, he was the Founding Chairman of the U.K. and Republic of Ireland Chapter of the IEEE Robotics and Automation Society, where from 1997 to 1999, he was the Chairman. He is an Associate Editor of the International Journal of Robotics and Autonomous Systems. During 2007, he was the General Chair of the International Conference on Intelligent Robots and Systems (IROS 2007). He is a Chartered Engineer. 